\title{
De l'interprétation systémique du projet de réhabiliter un bâtiment
}

\author{
Richard Cantin \\ Ecole Nationale des Travaux Publics de l'Etat, Université de Lyon \\ Rue Maurice Audin 60120 Vaulx-en-Velin, France \\ richard.cantin@entpe.fr \\ Jean-Claude Cryonnet \\ SyRHèse \\ 1005, route de Chapèze, 38300 Saint-Savin, France \\ jean-claude.cryonnet@syrhese.fr

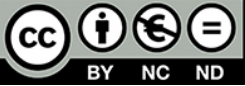

\section{Résumé}

Aujourd'hui, le projet de réhabiliter ne peut plus avoir simplement comme but de remettre en état un bâtiment. Il doit aussi transformer le bâtiment pour prendre en compte les nouvelles contraintes du développement durable, de la transition énergétique et du changement climatique. Ainsi, depuis plusieurs années, l'environnement des projets de réhabilitation se complexifie, et pour atteindre les niveaux les plus élevés de performances énergétiques et environnementales lors de la réhabilitation d'un bâtiment existant, il devient alors nécessaire de reconsidérer la complexité de l'opération de réhabilitation.

Dans ce contexte, le projet peut être réinterprété comme un système d'actions entreprises dans le but de transformer ou remettre en état le système bâtiment. Cette réinterprétation s'appuie ainsi sur les concepts de l'approche systémique et sur une représentation du générique de projet définie comme une combinatoire ayant présidé à l'invention du projet.

Les approches structurelle et fonctionnelle complètent la représentation du générique de projet en fournissant une vision distanciée et globale de la complexité du projet de réhabiliter, qui comme un système évolue selon une trajectoire combinant anticipation, césure et virtualisation.

Dans cet article, l'environnement complexe du projet de réhabiliter un bâtiment est présenté. Les différents concepts de l'approche systémique mise en œuvre pour faire face à la complexification du projet de réhabilitation sont précisés. Une chronique de la notion de projet permet ensuite l'élaboration d'une représentation du générique du projet. Enfin, cette représentation est complétée par les éléments d'une modélisation systémique visant à interpréter différemment le projet de réhabiliter.

\footnotetext{
Abstract

The target of retrofitting project only cannot be to retrofit a building. It must also modify the building integrating the new constraints of sustainable development, the energy model transition and climate change. Thus, for several years, the environment of retrofitting projects become more complex, and for reaching the highest levels of energy and environmental performance during the rehabilitation of an existing building, it is necessary to consider the complexity of the retrofitting action.

In this context, a project can be viewed as a system of actions in order to convert or refurbish the building system. This interpretation is based on concepts of the systemic approach and on a generic representation with a combination having presided at the project invention.

The structural and functional approaches provide a generic representation of a distanced and global view of the complexity of the retrofitting project, which as a system evolves along a path combining anticipation, caesura and virtualization.

In this paper, the complex environment of the retrofitting project is presented. Different concepts of the systemic approach used to deal with the complexity of the retrofitting project are described. A chronicle of project allows to elaborate a generic representation of project. Finally, this representation is complemented by elements of systemic modeling for differently interpreting the retrofitting project.
} 


\section{INTRODUCTION}

En France, le secteur du bâtiment émet plus de 20\% des émissions de gaz à effet de serre et consomme plus de $40 \%$ de l'énergie totale [1]. Avec un chiffre d'affaires de près de 130 milliards d'euros, c'est-à-dire deux fois plus élevé que celui des travaux publics, le secteur du bâtiment compte près de 1,5 million d'emplois [2]. Il est donc un secteur clé pour assurer la transition énergétique, réduire la dépendance d'une société sous le joug de l'approvisionnement énergétique et limiter les impacts environnementaux.

Le parc immobilier français n'augmentant que de 1 à $2 \%$ par an, les actions principales à mener passent par les opérations de réhabilitation. Ces opérations peuvent viser simultanément plusieurs objectifs comme réduire les factures énergétiques, augmenter le recours aux énergies renouvelables, améliorer le confort des occupants, assurer l'accessibilité et la sécurité des personnes, valoriser le patrimoine ancien, etc.

Cependant, lors de chaque opération de réhabilitation d'un bâtiment, plusieurs disciplines et métiers sont mobilisés. Plus de 40 corps de métiers peuvent être identifiés et la traditionnelle fragmentation de chaque opération en lots techniques distincts, relatifs au bâti (maçonnerie, isolation thermique, menuiseries extérieures, etc.) et aux équipements techniques (chauffage, plomberie, électricité, etc.) paraît de moins en moins opérationnelle lorsque le projet de réhabilitation vise une performance globale élevée pour le bâtiment existant.

Ainsi, pour atteindre les niveaux les plus élevés de performance dans un environnement complexe, il est nécessaire de reconsidérer le projet de réhabilitation à la lumière du paradigme systémique.

\section{L’ENVIRONNEMENT COMPLEXE DU PROJET DE RÉHABILITER}

\section{A. L'opération de réhabilitation}

La réhabilitation marque l'évolution du bâtiment, et désigne, dans cet article, l'amélioration du bâtiment existant, la remise en état historique (restauration) et la reconstruction (rénovation).

L'opération de réhabilitation compte trois temps, celui de la projection, de la réalisation et du bâtiment réhabilité (transformé) appelé à vivre par lui-même avec ses différents utilisateurs.

La performance recherchée par la réhabilitation s'élabore à partir des diverses contraintes existantes, des différents objectifs, niveaux et horizons de décision des acteurs. Cependant la performance globale d'un bâtiment existant est difficile à caractériser. En effet, la performance est une notion polysémique relative aux objectifs, aux résultats et aux actions, qui demande une vision distanciée et globale.

La multiplication des exigences entraine une complexification de l'environnement de la réhabilitation par une multiplication d'expertises, d'audits et de diagnostics : diagnostics d'experts (thermique, acoustique, éclairage, fondations, pathologies, etc.), diagnostics réglementaires (sécurité, amiante, plomb, termites, réglementation thermique, etc.), diagnostics de contrôle (amiante, plomb, termites, risques naturels et technologiques, performance énergétique, gaz, électricité), etc.

De nombreux retours d'expériences montrent les difficultés à maîtriser tous les éléments de la conformation complexe qu'est l'environnement de la réhabilitation. Les professionnels du secteur du bâtiment sont confrontés à l'accroissement de la complexité économiques, réglementaires, sociales, environnementales et au désarroi des mutations imprédictibles du secteur. Les modèles, les références et les organisations du passé, fortement marqués par le paradigme cartésien, ne suffisent plus à maîtriser les nouvelles opérations de réhabilitation complexes se voulant très performantes. Pour chaque opération, l'abondance des informations et des données, l'entrelacement des règlementations et des avis, et le foisonnement des multiples stratégies et représentations possibles de la réhabilitation deviennent en réalité difficiles à appréhender.

Ainsi depuis plusieurs années, l'environnement des projets de réhabilitation se complexifie, et assurer une garantie de performance, pour les opérations de réhabilitation, simultanément dans les domaines économiques, sociaux, énergétiques et environnementaux, apparaît bien incertain.

\section{B. Face à la complexification}

La complexité de la réhabilitation nait de la multiplicité et de la diversité des actions entreprises dans le but de remettre en état le bâtiment. Pour appréhender ces actions, non pas isolément mais globalement, en tant que parties d'un ensemble, le recours à l'approche systémique peut être envisagé.

Dans ce cadre, un bâtiment peut être considéré comme un système, c'est-à-dire comme un ensemble d'éléments en interaction dynamique organisé en fonction d'un but et évoluant dans des environnements. De la reconnaissance du grand nombre d'éléments différents et constitutifs du bâtiment naît alors le sentiment de complexité [3].

Plusieurs définitions du système mettent l'accent sur les notions d'interrelation et de totalité [4]. Il peut s'agir d'une unité globale organisée d'interrelations entre éléments, actions ou individus [5], ou d'un objet actif, stable et évoluant dans un environnement et par rapport à quelque finalité [6]. 
L'approche systémique fait appel à différents concepts spécifiques [7]. Par exemple, la globalité exprime l'interdépendance des éléments du système, la cohérence de l'ensemble et traduit la propriété du système où le tout est plus que la somme des parties. Le concept de globalité est complété par le concept d'interaction en s'intéressant au niveau de chaque relation au rapport d'influence et d'échange entre les éléments pris deux à deux [7].

L'organisation renvoie à l'agencement de relations entre les éléments. Elle produit une unité complexe dotée de qualités que n'ont pas chacun des éléments.

La finalité, dans le cas d'un système artificiel comme le système bâtiment, est définie par le concepteur. Le système est construit pour assurer des fonctions traduisant cette finalité. Les finalités correspondent à des buts visés, et non nécessairement atteints. Tout se passe comme si le système visait la satisfaction d'une ou plusieurs finalités.

Le système peut aussi être défini à partir des finalités, de ses fonctions et transformations, et de son environnement [8].

Décrit selon une approche structurelle, un système comprend quatre composants [4] :

国 Une frontière qui le sépare de son environnement et qui est plus ou moins perméable ;

四 Des éléments qui peuvent être identifiés, dénombrés et classés ;

四 Des réseaux de relation, de transport et de communication qui véhiculent des matières, de l'énergie, des informations ;

国 Des réservoirs dans lesquels sont stockés des matières, de l'énergie, des produits ou de l'information, indispensables au bon fonctionnement du système car, sans eux, celui-ci ne pourrait ajuster correctement son fonctionnement.

Décrit selon une approche fonctionnelle [4], le système comporte quatre composants:

网 Des flux de nature diverse (matières, produits, énergies, informations, etc.) qui circulent dans les différents réseaux du système ;

Des centres de décision qui reçoivent les informations et les transforment en actions;

Des boucles de rétroaction qui ont parfois pour objet d'informer les décideurs;

国 Des délais de réponse qui permettent de procéder aux ajustements dans le temps nécessaire à la bonne marche du système.

Les concepts de l'approche systémique permettent de proposer d'autres représentations, d'autres modélisations du système bâtiment. Ils aident à se détacher du paradigme cartésien aujourd'hui dominant dans la perception et la représentation du bâtiment. Les approches structurelle et fonctionnelle, venant compléter l'approche cartésienne, aident à reconsidérer la réalité de l'ensemble du système bâtiment, ses finalités, sa globalité et son organisation. L'approche systémique permet alors de visualiser le bâtiment et ses occupants dans et en interaction avec les environnements économiques, sociaux et environnementaux.

\section{LE PROJET DE RÉHABILITER, LIEU DE RÉINTERPRÉTATION}

Roger Aïm [9] tient pour inventeur du projet Filippo Brunelleschi, ingénieur-architecte et peintre italien de la Renaissance, réalisateur du célèbre Duomo de Florence. Cette invention n'a lieu qu'en réalisant une combinatoire entre trois éléments :

困 Le premier d'ordre psychologique, puisqu'il s'agit du souci d'anticipation;

氾 Le deuxième est d'ordre de la technique (artefact) de l'ordre de la virtualisation [10];

[e troisième est d'ordre historique et social, de l'ordre de la césure.

Au besoin d'anticipation et d'adaptation à l'existant (le corps du bâtiment étant déjà conçu et construit), Filippo doit répondre par une préparation minutieuse, une description par avance des modalités de réalisation : il faudra décrire l'ouvrage, c'est-à-dire autant la façon de faire que le résultat, dans un descriptif indissociable.

Mais, pour se faire comprendre dans un monde où la langue et l'écrit ne sont pas maitrisés, il faut passer par la représentation graphique, picturale ; c'est donc dans la mise au point d'une technique, d'un artefact, permettant la virtualisation dans l'espace qu'il va chercher et trouver, la perspective. Cette doublette inventive va trouver son milieu historique et social dans la césure tout autant volontaire,(ne plus gérer le chantier, se consacrer à la conception) que subit (division du travail, spécialisation, éloignement géographique) entre les métiers, les lieux, les moments de la fabrication et de la conception rendus distants par le développement du transport et du commerce. 
Il convient de ne pas ignorer ce générique ${ }^{1}$ pour bien mesurer sa contingence (Figure 1). La césure temporelle et géographique est-elle la même à l'heure de la communication accélérée, du drone ? La virtualisation est-elle la même à l'heure de la simulation informatique? La puissance d'anticipation est-elle la même avec des capacités statistiques immenses ?

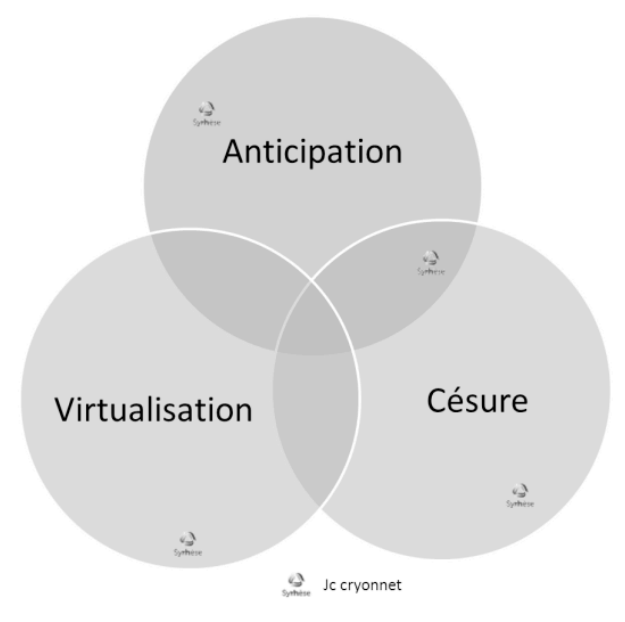

Figure 1. Représentation du générique du projet [12]

On laissera ici l'histoire et l'évolution de la notion pour reprendre la chronique du projet en ce début de $21^{\text {ème }}$ siècle et analyser la définition et la pratique qui en sont faites dans trois secteurs économiques, celle du bâtiment, de l'industrie manufacturière, et de l'informatique.

Si le monde du bâtiment a vu naître le concept de projet, pour mieux décrire l'ouvrage, l'industrie naît avec le concept d'opération, si le chantier est un lieu de co-activité, la chaîne est un lieu de tâches successives avec des activités divisées, hiérarchisées. L'industrie désintègre l'ouvrage, le travail est détaché de son résultat, l'acte et le produit sont séparés pour éclater la production en une chaîne. C'est, face aux limites de cette organisation linéaire, hiérarchique et arborescente, qu'on réinvente une organisation par projet, transverse et matricielle. Cette réinvention, ne reprend cependant pas le générique du projet, car elle installe une structure de gestion concurrente de la structure hiérarchique, mais qui ignore encore la complexité de la notion d'ouvrage. L'électro-mécanisation de la fabrication, va surajouter à la division œuvre-travail ainsi apparu, la séparation homme/système-machine.

L'informatique va amener une autre notion du projet puisqu'il s'agit de faire faire, non à des hommes mais à la machine, un ordinateur, par des opérations logiques successives ; la césure est ici homme-machine et l'écriture de la commande va demander l'invention des langages, l'organisation de différents niveaux de déclinaison de l'interface entre le milieu environnant et le cœurcalculateur binaire. Le développement d'une interface efficace entre homme et machine, va en fait créer un système « hommemachine ».

Une profusion d'outils, de méthodes et surtout un lancement de création de nouveaux artefacts informationnels, agents intelligents, réalisés par modules échangeables, va nécessiter une remise en ordre, en ligne de toutes sortes de développement de programme. Chaque projet, pris au sens de faire faire quelque chose par un artefact informatique, nécessite une organisation des projets et des ressources mais, de leur foisonnement, de l'appartenance possible d'un module à plusieurs projets, va naître, non plus la gestion de projet mais la gestion des projets, de portefeuilles de projets à aligner.

Le bâtiment, de son côté, va être perméable, dans la première partie de l'après-guerre à la vision industrielle dans la phase de réalisation, puis dans la deuxième partie, à la vision informatique dans sa phase de conception et de gestion.

Aujourd'hui, le projet situe donc sa molécule générique (Figure 1) dans une espace compris entre division et concourrance, coordination et différenciation, standardisation et variété, multiplicité et alignement, modélisation et langage, différence et répétition.

\footnotetext{
${ }^{1}$ Le générique est vu ici comme un lieu, un espace de superposition de connaissances et de pratiques [11].
} 


\section{Acta Europeana Systemica $n^{\circ} 4$}

Les outils de gestion de projet s'appuient sur un découpage mécanique, en tâches et sous-processus, reliés par des échanges économiques de type clients-fournisseurs, issus de théories sociologiques de spécialisation et de division du travail, puis opposant l'obéissance et n'examinant pas la conception. Une sociologie basée sur l'imitation, un examen des phases de conception et une économie de l'échange du surplus permettraient d'ouvrir les outils de gestion de projet à une décomposition systémique des faits, à une économie des connaissances mises en jeu.

Ces outils, où les éléments sont cloisonnés, mettent en exergue une progression linéaire de la conception et de la fabrication, n'admettant comme gestion complexe du temps qu'un éventuel parallélisme des tâches, conduit à une cession séquentielle du projet, un découpage du produit, où chaque étape est bornée par une décision assez binaire d'acceptation ou de refus. Le temps est, dans cette approche, une quantité (de durée d'exécution d'une tâche, de travail) alors qu'il pourrait être un espace plus qu'une unité de mesure.

Les modèles mécaniques du projet sont encore nombreux, enchaînements pauvres, liaisons temporelles, paramètres restreints (temps, coût, qualité), le troisième (qualité) étant un fourre-tout, au mieux une valorisation des exigences posées par une analyse fonctionnelle du besoin qui permettra une analyse de la valeur du produit, au pire une expression qui marque difficilement une méconnaissance de l'ouvrage ${ }^{2}$, le travail à réaliser étant le point aveugle des méthodes de gestion de projet des écoles de management.

Les outils de traitement de l'information et de la communication, comme la densification des interactions réinterrogent les trois fondamentaux du générique (Figure 1). La virtualisation s'étend à la réputation par la certification, à la mise en œuvre de contrats de plus en plus sophistiqués, à la simulation informatique. La césure temporelle et physique se réduit grâce à la rapidité des communications et la capacité de contrôle et de visualisation à distance, l'anticipation devient une exigence de plus en plus forte avec l'exigence d'assurance des personnes, des biens et des retours sur investissement. Elle trouve des moyens toujours accrus dans des capacités de traitements statistiques plus performantes. La césure entre les disciplines et métiers s'estompent grâce à la capacité d'adaptation plus grande des acteurs, et aussi à une préfabrication croissante, à une interopérabilité des matériels comme des informations.

Le projet de réhabilitation du bâtiment est bien un lieu de réinterprétation de ces fondamentaux.

\section{ELÉMENTS D’UNE MODÈLISATION SYSTÉMIQUE DU PROJET DE RÉHABILITER}

La réinterprétation du projet de réhabilitation peut passer par différents modèles développés selon l'approche analytique ou systémique.

Mais si nous ne raisonnons qu'à partir de modèles, il est alors essentiel de s'interroger sur la manière dont sont élaborés les modèles sur lesquels nous raisonnons [13]. Ils sont des représentations artificielles des situations qui fournissent les éléments d'interprétation.

Le recours à des représentations mathématiques du projet de réhabilitation peut répondre aux besoins de rationalisation. Mais la réalité du projet de réhabilitation, lieu de réinterprétation, n'est pas toujours rationnelle. L'approche analytique ne fournit pas les réponses aux insuffisances d'un modèle rationnel qui voudrait maîtriser totalement la complexité du projet de réhabilitation. La contingence du projet de réhabilitation d'un bâtiment existant est liée à la présence de facteurs variés (physiques, humains, etc.), plus ou moins aléatoires, imprécis voire inconnus. Elle ne permet pas de proposer un modèle mathématique ou analytique représentant en détail ou avec exactitude le projet de réhabilitation.

L'approche systémique fournit les éléments d'une vision distanciée, organisée et globale du projet de réhabilitation. Le projet de réhabiliter peut être caractérisé selon une approche structurelle :

困 La frontière du projet de réhabilitation se définit selon la ou les finalités de la réhabilitation, selon le ou les diagnostics. Chaque diagnostic fournit une frontière pour le projet. La délimitation de l'audit (ou du diagnostic) relève en partie de la subjectivité de l'auditeur (ou du diagnostiqueur). Par exemple, le diagnostic énergétique d'un bâtiment exige une visite où les limites de l'investigation (anticipation) associées aux facteurs humains constituent une frontière dans le recueil des informations sur le comportement thermique du bâtiment existant ;

\footnotetext{
${ }^{2}$ Ouvrage : terme qui désigne le produit d'un travail réalisé selon un processus et des modalités précises garantes du résultat, de sa solidité, maintenabilité, etc. Le terme définit à la fois le résultat et le travail pour y arriver, ce qui différencie ouvrage et produit, maitre d'ouvrage et client. Un tricot n'est pas un pull-over, une excavation n'est pas un trou...
} 
困 Les éléments du projet sont hétérogènes (césure) comme par exemple le bâtiment et ses composants, les diagnostics, les données recueillies, les moyens mis en œuvre, les objectifs, les personnes, les méthodes de gestion, l'échéancier, les coûts ;

龱 Un réseau de relations et de communication assurent la circulation des flux d'information, des flux financiers, des flux de personnes, des flux de matière et d'énergie au sein du projet. Depuis l'origine du projet, en passant par sa planification, l'exécution des tâches et le contrôle jusqu'à sa clôture, différents réseaux matériels et immatériels peuvent être identifiés entre les éléments (virtualisation);

困 Des réservoirs dans lesquels sont stockés les éléments et les informations sont indispensables au bon fonctionnement du système projet. L'archivage, les différentes formes de mémorisation des données, les représentations (rapports, plans sur différents supports) sont ces réservoirs (virtualisation).

Cette représentation structurelle peut être complétée par les aspects fonctionnels :

国 La complexité d'un bâtiment existant ne permet pas une connaissance exhaustive des flux (virtualisation). Les différents audits du bâtiment existant produisent des informations mais les limites et les imprécisions de l'investigation les rendent incomplètes et incertaines. Les acteurs fournissent des informations produites selon différents points de vue (anticipation). Il en résulte plusieurs représentations d'un même bâtiment (césure) qui impacte les capacités d'anticipation de chacun;

网 Les acteurs du projet constituent des centres de décision du système projet. Ils doivent travailler avec des volumes importants de données qui couvrent plusieurs disciplines. Le recours à des approches simplificatrices conduit à des prises de décision qui ne sont pas optimisées (césure, biais d'anticipation);

国 Les boucles de rétroaction du système projet sont identifiées, par exemple, avec des infirmations d'hypothèses en cours de chantier, des réactions du bâtiment suite aux interventions prévues. Elles proviennent également des retours d'expériences relatifs aux précédentes opérations de réhabilitation sur le même bâtiment ou à des réhabilitations sur des bâtiments similaires (études comparatives, benchmarking, simulations, etc.). Elles permettent aux acteurs de vérifier et valider leurs modèles de décision (anticipation, virtualisation). Le caractère unique de chaque réhabilitation est cependant une difficulté majeure à l'identification de solutions de réhabilitation qui seraient universelles (césure) ;

四 Les délais de réponse sont identifiables pour chaque acteur impliqué dans le projet. La gestion du temps est l'un des paramètres importants de l'organisation et de la dynamique du projet de réhabilitation. Le caractère irréversible du temps donne une valeur particulière au projet, y compris pécuniaire (césure).

L'approche systémique conduit à définir le projet de réhabiliter comme un ensemble d'actions entreprises dans le but de remettre en état le bâtiment et de prolonger sa durée de vie dans différents environnements. Ces environnements multi-physiques et multi-échelles sont énergétiques, climatiques, économiques, sociaux, etc. Le projet est un système ouvert sur ces environnements avec lesquels il échange des flux de matière, d'énergie et d'information. L'ensemble d'actions doit répondre à des besoins définis dans des délais fixés, dans la limite d'un budget alloué. Compte tenu de la complexité d'un bâtiment, le projet de réhabilitation est marqué par l'interdisciplinarité qui appelle une intelligence collective.

Le projet évolue comme un système selon une trajectoire. Chacun de ses éléments apporte sa propre dynamique, ses propres objectifs, et participe à une transformation des finalités de la réhabilitation. En cela, ils contribuent à une évolution des outils de mesure et des performances à atteindre. La maitrise de la trajectoire du projet nécessite l'association de tous les composants du système, de toutes les compétences des acteurs. Dans ce cadre, les compétences des acteurs ne peuvent pas être évaluées indépendamment les unes des autres, ni classées hiérarchiquement. Leur diversité assure la différenciation puis la coordination du projet de réhabilitation [8]. Cette trajectoire, marquée d'incertitudes décisionnelles (césure) et d'incomplétudes informationnelles (virtualisation), fait du projet de réhabilitation un système décisionnel (anticipation) et non un système programmé (prévision). Le leader du projet guide alors le système avec la coopération des personnes participantes au projet, chacune constituant un centre de décision influençant la trajectoire du système projet.

Enfin, si l'approche systémique conteste et complète l'approche analytique, sans l'exclure, elle ne peut pas prendre en compte tous les paramètres entrant dans les mécanismes décisionnels, laissant le champ libre à des instabilités (césure) que le chef de projet devra assumer.

\section{CONCLUSION}

Les changements énergétiques et environnementaux annoncés pour le $21^{\text {ème }}$ siècle imposent une évolution du secteur du bâtiment, et notamment une réinterprétation de la notion du projet de réhabilitation. La réinterprétation systémique doit répondre à 


\section{Acta Europeana Systemica $n^{\circ} 4$}

la complexification croissante du secteur du bâtiment et de ses environnements. Le projet de réhabiliter doit intégrer une complexité accrue par les exigences énergétiques et environnementales plus fortes et une dynamique décisionnelle caractérisée par son instabilité et son interdisciplinarité.

Cette réinterprétation de la notion de projet peut s'appuyer sur l'invention du projet par Filippo Brunelleschi issue d'une combinatoire entre trois éléments : l'anticipation, la virtualisation et la césure.

Cette réinterprétation rompt avec le paradigme d'une programmation traditionnelle, linéaire et séquentielle, qui sous-estime la complexité d'une phase d'évolution anachronique du système bâtiment. Cette rupture inévitable interroge les compétences requises et la formation du responsable de projet en réhabilitation [14].

Dans ce contexte, il apparaît alors indispensable d'explorer l'évolution des activités de programmation d'opérations dans le secteur du bâtiment, pour les nouvelles opérations de réhabilitation, à la lumière du paradigme systémique, en revisitant les notions de processus, de programme et de « système projet».

Le projet de réhabiliter un bâtiment ne réclame donc plus un gabarit du passé à réappliquer, mais un modèle à construire comme la représentation locale, historique, et ad hoc, du système d'actions qui se situe à l'intersection de plusieurs systèmes : systèmes techniques du bâtiment, systèmes productifs des acteurs, systèmes patrimoniaux des actifs immobiliers, systèmes urbains environnants, systèmes sociaux, systèmes utilisateurs, systèmes juridiques des contrats et responsabilités.

\section{REFERENCES}

[1] MEDDE. Ministère de l'Ecologie, du Développement Durable et de 1'Energie, France. Site web consulté 2014. http://www.developpement-durable.gouv.fr/Batiments-existants-.html

[2] FFB. Fédération Française du Bâtiment. Le bâtiment en chiffres. 2014

[3] Atlan, H. Entre le cristal et la fumée. Le Seuil. Paris. p79. 1979.

[4] Durand, D. La systémique. Paris, PUF, 126p. 1994.

[5] Rosnay, de J. Le macroscope, vers une vision globale. Paris, Seuil, 346p. 1975.

[6] Morin, E. La méthode, la nature de la nature. Paris, Seuil, 399p, 1977.

[7] Donnadieu, G. Durand, D. Neel, D. Nunez, E. et Saint-Paul, L. L'approche systémique : de quoi s'agit-il ? Site web consulté juillet 2014. Afscet http://www.afscet.asso.fr/SystemicApproach.pdf

[8] Le Moigne, J.L. Théorie du système général. Vendôme, PUF, 126p, 1994.

[9] Aïm, R. Filippo Brunelleschi. Le dôme de Florence. Paradigme du projet. Edition Hermann, 2011.

[10] Levy, P. Sur les chemins du virtuel. Site web consulté Juillet 2014. http://hypermedia.univ-paris8.fr/pierre/virtuel/virt0.htm

[11] Mathieu, N. et Schmid, A.F. Modélisation et interdisciplinarité. Six disciplines en quête d'épistémologie. Coédition Quæ/NSS-Dialogues, Coll. «Indisciplines », 348 p. 2014.

[12] Cantin, R. et Cryonnet, J.C. Le projet de réhabilitation d'un bâtiment vu comme un système. 9th Congress of EUS-UES European Union for Systemics. Valencia, 2014.

[13] Le Moigne, J.L. La modélisation des systèmes complexes. Afcet Systèmes. Dunod. Paris. 170p.1995.

[14] Clénet, J. et Poisson, D. Coord. Complexité de la formation et formation à la complexité. L'Harmattan, Paris, 286 p. 2007. 
